\title{
Mechanotransduction mechanisms for coordinating uterine contractions in human labor
}

\author{
Roger C Young ${ }^{1,2,3}$ \\ ${ }^{1}$ PreTel, Inc., Memphis, TN, USA, ${ }^{2}$ University of Newcastle, Newcastle, Australia and ${ }^{3}$ Imperial College of London, \\ Chelsea and Westminster Hospital, London, UK
}

Correspondence should be addressed to R C Young; Email: ryoung@pretelhealth.com

\begin{abstract}
This review presents evolving concepts of how the human uterus contracts in pregnancy, with emphasis on the mechanisms of long-distance signaling. Action potential propagation has historically been assumed to be the sole mechanism for signaling and tissue recruitment over both short and long distances. However, data in animals and humans indicate that a single action potential does not travel distances greater than a few centimeters. To address this enigma, a long-distance signaling mechanism based on hydraulic signaling and mechanotransduction is developed. By combining this mechanism for long-distance signaling with the action potential propagation mechanism for signaling over short distances, a comprehensive dual mechanism model (or 'dual model') of uterine function is formulated. Mechanotransduction is an accepted phenomenon of myometrium, but the dual model identifies mechanotransduction as relevant to normal labor. For hydraulic signaling, a local contraction slightly increases intrauterine pressure, which globally increases wall tension. Increased wall tension then mechanically induces additional local contractions that further raise pressure. This leads to robust, positive feedback recruitment that explains the emergence of consistently strong contractions of human labor. Three key components of the dual model - rapid long-distance signaling, mechanical triggering, and electrical activity converge with the concept of mechanically sensitive electrogenic pacemakers distributed throughout the wall. The dual model retains excitation-contraction coupling and action potential propagation for signaling over short distances $(<10 \mathrm{~cm})$ and hence is an extension of the action potential model rather than a replacement.

Reproduction (2016) 152 R51-R61
\end{abstract}

\section{Introduction}

A great deal is known about how uterine cells contract, but there is a large gap of understanding about how billions of myocytes coordinate individual contractions into an organ-level contraction of labor (Lammers 2013). With the exception of consciousness and self-awareness of the brain, the laboring uterus is the only organ in the human body lacking a generally recognized, well-supported mechanism for primary function. The uncertainty has significant consequences, since it is likely that the development of new methods to modulate human labor has been hindered by lack of a comprehensive model for generating uterine contractions. Additionally, this topic represents a major gap in the knowledge base of practicing obstetrical providers, which perhaps contributes to adverse pregnancy outcomes of individuals.

This paper reviews several models of uterine function, and emphasizes the evolution of the concepts of organlevel signaling. Perhaps because of the analogy with heart function, action potential propagation has been widely believed to be the only mechanism utilized for recruiting tissue in a normal uterine contraction (Smith et al. 2015). But discrepancies arise when investigating action potential signaling over long distances, which suggests

(C) 2016 Society for Reproduction and Fertility

ISSN 1470-1626 (paper) 1741-7899 (online) that other mechanisms are needed. The newest model of global uterine function combines fluid mechanics and mechanotransduction for long-distance signaling with action potential propagation for short-range signaling. This dual-mechanism model, or 'dual model', is consistent with animal and human data on long-distance uterine signaling. Earlier versions of the dual model have previously been presented as mathematical simulations (Young \& Barendse 2014, Young 2015).

\section{Limitations}

The topic of this review is uterine contractions during pregnancy, when the uterus is a pressurized organ. It does not address contractions in either the nonpregnant state or very early pregnancy when the uterus is not pressurized. A review is inherently subjective. Some models and mechanisms that are not well supported or were proven inaccurate are not included. Many excellent researchers and coworkers of those mentioned who made major contributions to the field have not been credited because of space limitations. Single quotation marks are used to emphasize specific terminology used by the original authors when defining their model. 


\section{Intrauterine pressure and the need to globally coordinate uterine contractions for effective labor}

Although the quintessential endpoint of labor is cervical dilation, it is critically important that the uterus generate large intrauterine pressures for dilation to occur. Alvarez and Caldeyro-Barcia were the first to reliably measure intrauterine pressure rises caused by human uterine contractions (Alvarez \& Caldeyro 1950). CaldeyroBarcia went on to devise the 'Montevideo unit' for quantifying intrauterine pressure (Caldeyro-Barcia et al. 1957), and related Montevideo units to the progress of labor. The concept of assessing intrauterine pressure is still relevant and clinically useful for determining the adequacy of uterine contractions.

Jeffcoate was possibly the first to consider the importance of coordinating uterine contractions at the organlevel (Jeffcoate 1948). Using clinical reasoning, he proposed that the contractions of 'false labor' were 'incoordinate'. Alvarez and Caldeyro-Barcia tested the concept of uterine coordination by measuring local contractile activity in the human uterus first with an early multichannel uterine EMG (Alvarez \& Caldeyro 1950), then with small balloons placed transabdominally into the uterine wall (Caldeyro-Barcia \& Poseiro 1959). While limited to using only three or four sensors at a time, these studies supported Jeffcoate's proposal, concluding that 'pre-labor' contractions are different from labor contractions because of poor coordination.

Csapo, a contemporary of Caldeyro-Barcia, was also a leader in the field. He described a 'transient' condition that is similar to Caldeyro-Barcia's concept of pre-labor contractions. To explain the weakness of the contractions that occur before labor, Csapo proposed 'local contractions' that do not encompass the majority of the uterine wall (Csapo 1970a,b). He reasoned that the areas of the uterine wall that were not participating in a contraction would elongate when the actively contracting areas increased wall tension, and elongation of the passive areas would attenuate the rises of pressure. Because of this effect, high pressures of labor can be generated only when most of the uterine wall is contracting at the same time in a coordinated manner.

Csapo did not explicitly discuss uterine shape change as an important factor in modulating pressure development, and perhaps over-emphasized the 'all-ornothing' nature of the contractions of excitable smooth muscle, but together with organ-level coordination, these factors largely determine the relationship between contractions of individual myocytes and generation of intrauterine pressure. He did describe how to apply Laplace's Law to provide a quantitative relationship between intrauterine pressure, wall tension, wall thickness, and the local radius of curvature (Csapo 1970a).

In summary, from Caldeyro-Barcia, we learned that frequent contractions that consistently generate high intrauterine pressures are necessary and sufficient for labor. From Csapo, we understand that in order to achieve the high pressures, the uterus must somehow contract most areas at the same time.

\section{Electrical activity, propagation of the action potential, and a uterine contraction}

Caldeyro-Barcia described his 'triple descending gradient' model of uterine function as a wave-like contraction which starts at the fundus, spreads down the uterus, and decreases in strength and duration as it progresses (Alvarez \& Caldeyro 1950). The fact that uterine muscle creates bioelectrical activity as it contracts was established at the time, although Caldeyro-Barcia did not emphasize that contractile activity occurs as a direct result of electrical activity.

Detailed investigations into the electrical properties of uterine cells and tissue began in the mid-1950s, and the concept of excitation-contraction coupling in myometrium was soon formalized (Csapo \& Suzuki 1957, Csapo 1959, Mironneau 1973). Recent advances have comprehensively elucidated the relationship between plasma membrane excitability properties and intracellular mechanisms that leads to cellular contractions (Wray et al. 2015).

In 1963, Csapo and Takeda (Csapo \& Takeda 1963) reported that when in active labor, uterine bioelectrical signals were expressed in 'synchrony' with the generation of intrauterine pressure. Using rabbit data to create a model and then testing the predictions of the model in human, Csapo proposed that local contractions are created by localized bioelectrical discharges of the myometrium (Csapo \& Takeda 1965). He defined contractions that remained local as 'nonpropagating' and labor contractions as 'propagating' (Csapo 1970a). These terms were likely chosen to emphasize that his concept for all uterine signaling involved action potential propagation.

Connexin 43 is the protein responsible for cell-tocell electrical coupling in the heart, and Garfield's discovery of connexin 43 in the uterus (Garfield et al. 1977) provided a mechanism for uterine electrical communication. Through the 1980s and 1990s, emerging data supported the concept that connexin 43 increases myometrial excitability, and inferred that action potential propagation is the mechanism for both short- and long-distance electrical signaling (Garfield et al. 1995).

Through the end of the twentieth century, action potentials were thought to perform three important functions: initiating and maintaining cellular contractions, recruiting cells for participation in the contraction, and organ-level signaling to coordinate contractile activity in all parts of the uterus. Yet discrepancies arose when experimentally testing action potential propagation as the mechanism for signaling long distances. 


\section{Problems with action potential propagation as a mechanism for tissue recruitment over long distances}

Tissue recruitment occurs during the rising phase of a contraction, which lasts approximately $20 \mathrm{~s}$ in human. There are two ways that a single propagating action potential can signal long distances within that time. First, a slowly moving action potential can travel in a straight path. Assuming the starting point is on the front wall, travel to the back wall is a distance of about $40 \mathrm{~cm}$. Thus, speeds greater than $2 \mathrm{~cm} / \mathrm{s}$ would be sufficient to accomplish this, and these values are routinely observed in human (Rabotti et al. 2010, Rabotti \& Mischi 2015) and rodent (Lammers 2013). However, there is a significant doubt that myometrial action potentials travel in straight paths. In sheep (Parkington et al. 1988), rodent (Lammers et al. 2008), and human (Mikkelsen et al. 2013, Rabotti \& Mischi 2015) action potentials are not found to travel linearly. The highest resolution electrical mapping of myometrium is in the guinea pig, which reveals that action potentials diverge and loop through tortuous paths, self-terminate, and usually do not propagate more than a few centimeters (Lammers et al. 2008) (Fig. 1). In their recent review, Rabotti and Mischi (2015) concluded that electrical propagation in human is unpredictable and demonstrates complex patterns.

The second way a single action potential can accomplish global recruitment is by traveling tortuous paths very rapidly. In the laboring uterus, very fast speeds have been reported, even exceeding $80 \mathrm{~cm} / \mathrm{s}$ (Lucovnik et al. 2011). These values have been questioned (Rabotti et al. 2011), however, since a technique using high-resolution EMG (64 electrode grid, $4 \mathrm{~mm}$ spacing) was required to clearly resolve propagating action potentials, and speeds measured during active labor were less than $4 \mathrm{~cm} / \mathrm{s}$ (Rabotti et al. 2010).

Additionally, an action potential propagation mechanism implies that tissue recruitment is contiguous and occurs without skipping over large areas. Using a 152 element array of superconducting quantum interference devices (SQUID), Ramon and coworkers (2005) found that localized biomagnetic activity (and by direct inference, bioelectrical activity) appeared suddenly in random locations that were not contiguous or anatomically related to each other (Fig. 2). The size of individual hot spots in the SQUID array was consistently found to be between 6 and $10 \mathrm{~cm}$. If the myometrium within the localized activities is recruited by action potential propagation, this suggests that the maximum distance an action potential can propagate is $6-10 \mathrm{~cm}$.

Hence the enigma - action potentials appear to propagate slowly in tissue, in complex pathways, noncontiguously, and over only short distances. This makes it difficult to envision how global recruitment can occur within $20 \mathrm{~s}$. Furthermore, the mechanism needs to be highly reliable, since strong contractions must be repetitively generated for effective labor. If signaling long

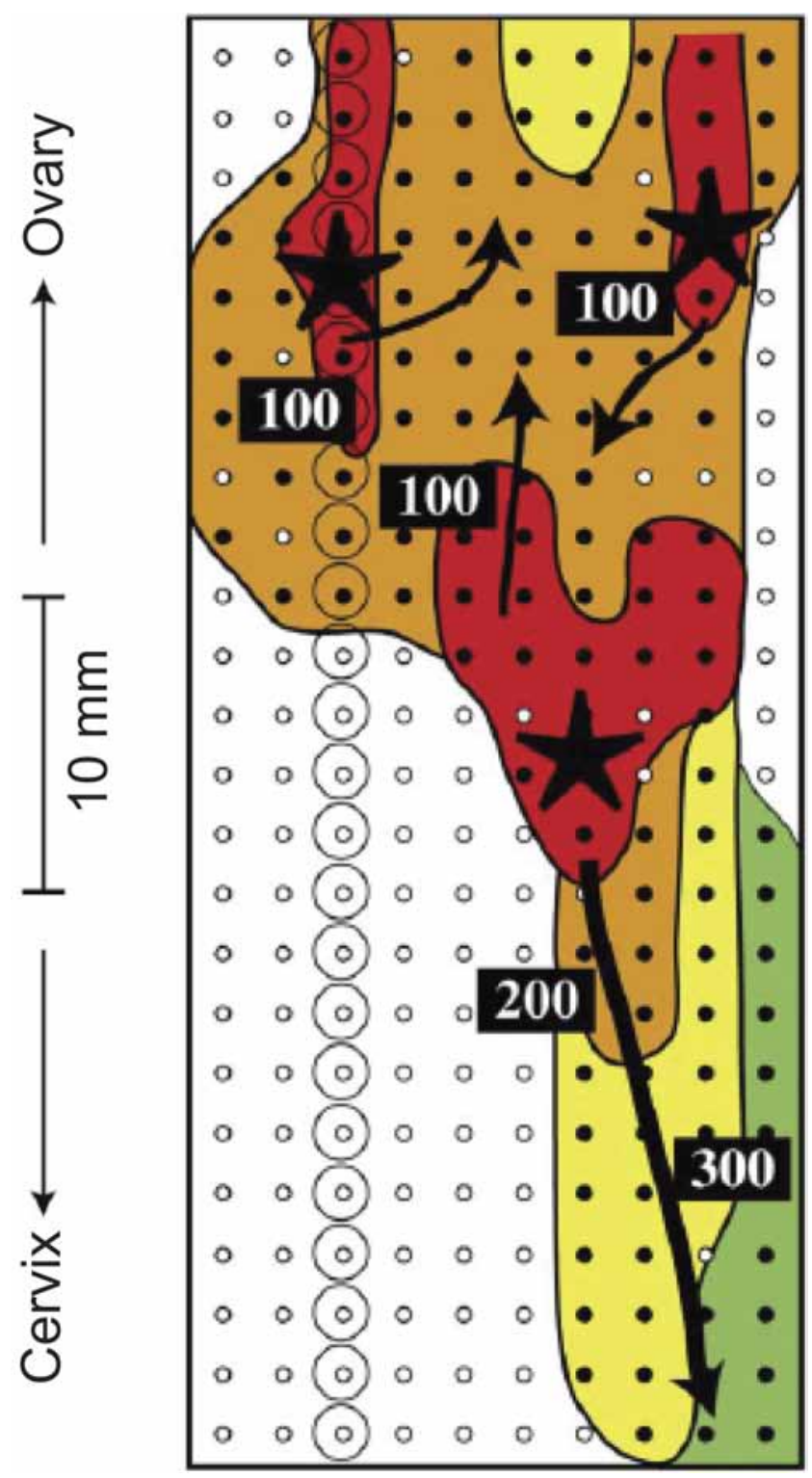

Figure 1 High density mapping of electrical activity of a pressurized guinea pig uterus, from Lammers (Lammers et al. 2008). Four action potentials originate nearly simultaneously from three locations (stars). Arrows represent the paths of different action potentials. Three action potentials within a few millimeters of each other travel in different directions.

distances by propagation of a single action potential seems unrealistic, then it is reasonable to consider other mechanisms.

\section{Mechanotransduction for organ-level signaling}

In 1947, Bozler (1947) reported that distention of the renal end of a dog ureter resulted in changes in electrical potential and a contraction that propagated down the ureter. Even though the ureter is not pressurized, this was the clear evidence that a visceral smooth muscle utilized 

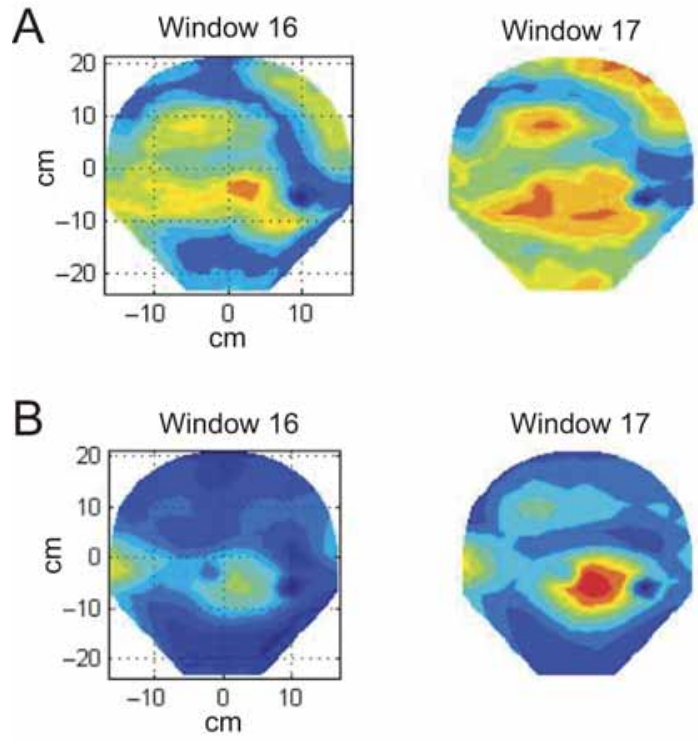
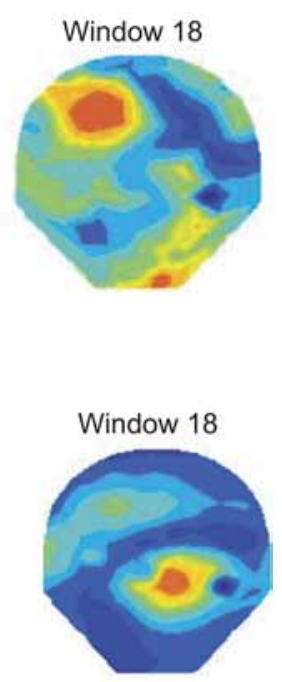

Figure 2 SQUID array images, adapted from Ramon and coworkers (Ramon et al. 2005). The patient is experiencing pre-labor contractions. Timedependent spatial representation from 152 sensors. Windows represent $20 \mathrm{~s}$ of averaged data. Each window overlaps with the previous and subsequent windows by $10 \mathrm{~s}$. The contraction peak occurs at window 17. (A) Data analyzed using a frequency synchronization technique, which compares signals from surrounding SQUID sensors. (B) Data analyzed measuring the power of individual sensors. In either method of analysis, hot spots of myometrial activity are seen. In both A and B, islands of activity appear without activation of neighboring tissue. Linear propagation of activity is not seen. mechanotransduction to express normal physiology. However, mechanical mechanisms for expression of normal uterine function have been investigated only sporadically.

The first experimental report to indicate mechanotransduction might be important in organ-level signaling for the uterus appeared in 1965 by Takeda, who studied the repressurized postpartum rabbit uterus (Takeda 1965). In this clever experiment, the electrical activity of two halves of a uterine horn was synchronized solely by mechanotransduction operating through 'hydrodynamic' signaling and intrauterine pressure (Fig. 3). Despite the likelihood that the experiments were performed in Csapo's lab, Csapo's name did not appear with Takeda's on the hydrodynamic signaling publication. Additionally, Takeda's publication was not included in Csapo's 1970 review of the field (Csapo 1970a,b), although Csapo did describe how hydrostatic effects can be applied to the uterus using Laplace's Law. In 1979, Wolfs and van Leeuwen (Wolfs \& van Leeuwen 1979) reviewed the field again. With 222 references dating as far back as 1871, their manuscript also failed to include Takeda's paper, and did not mention Csapo's analysis of uterine hydrostatics using Laplace's Law. It is not clear if subsequent research efforts were adversely influenced by the apparent lack of support for the model by leaders in the field, but it would be almost two decades before another report appeared that tested the hydrodynamic model.

In 1984, Sigger and coworkers (1984) studied a $3 \mathrm{~cm}$ tissue flap partially isolated in situ from the uterus of pregnant sheep. They found that electrical continuity was required for contraction of the flap to coincide with the uterine contraction, and Takeda's hydrodynamic mechanical mechanism, in particular, did not seem necessary. These results supported a model that utilized action potential propagation for all uterine signaling, and contributed to mechanotransduction being considered irrelevant to normal labor.
However, the same group, again using the pregnant sheep model, came to the opposite conclusion 4 years later when longer distances were considered (Parkington et al. 1988). Here, they measured uterine electrical activity from multiple surgically implanted electrodes separated by distances that varied from 1 to $16 \mathrm{~cm}$ and found that electrical activity rarely propagated more than $3 \mathrm{~cm}$ (Fig. 4). While unable to resolve the mechanism of signaling used to coordinate contractile activity over distances greater than $3 \mathrm{~cm}$, they speculated on the participation of an 'intrauterine fluid wave', and specifically noted that this mechanism was previously suggested by Takeda. For unclear reasons, the questions raised by this second paper were not immediately investigated in detail. For the next two decades, with only a few exceptions, little thought was given to mechanotransduction.

Barclay and coworkers (2010) published a mathematical simulation of global uterine function that was general enough to include mechanotransduction as a signaling mechanism. They assumed that a local contraction could directly initiate other local contractions, but limited the interactions to physically adjacent tissues. This formulation overcomes the problem that action potential propagation distances are limited, but because only near-neighbor interactions were included, the long-distance signaling properties were similar to those of long-distance action potential propagation. As a result, inconsistencies with empirical data persist.

\section{Uterine electrogenic pacemakers}

The nature and role of uterine pacemakers have long been a source of controversy (Lammers 2013). Fundamental physiological questions remain unanswered (Rabotti \& Mischi 2015), including the number of pacemakers the 
A

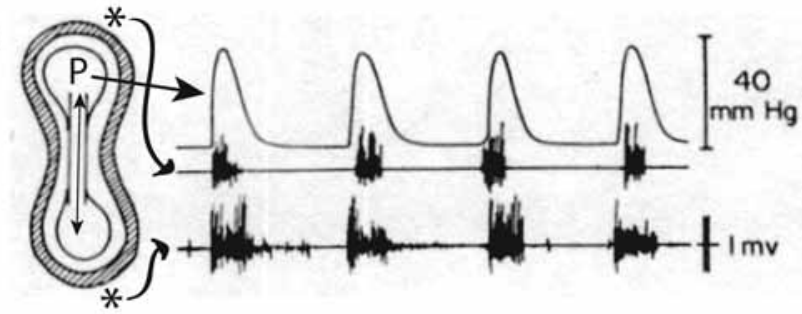

B

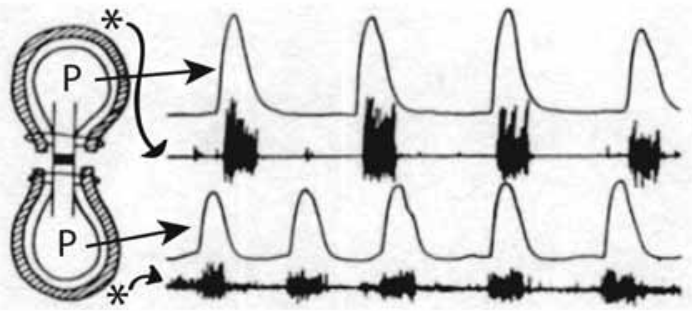

C

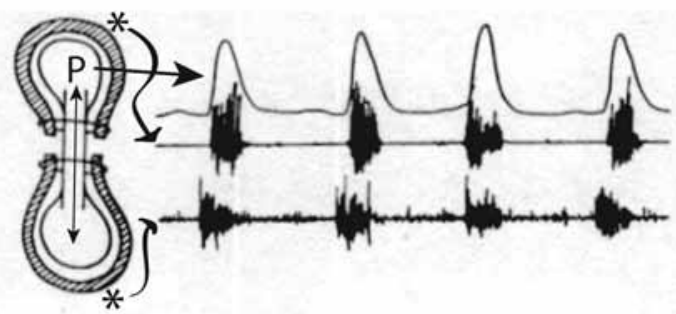

Figure 3 Hydrodynamic signaling in the rabbit uterus, adapted from Takeda (1965). Two balloons, hydraulically connected with a rigid tube, are placed within a rabbit uterus. Wire electrodes record EMG signals over each balloon, and the pressure within each balloon is also measured $(*$ indicates the approximate location for each electrode). (A) Continuity of tissue, free exchange of pressure between balloons results in generation of a single pressure. Synchronized activity is observed among pressure and both electrodes. (B) Tissue is cut between balloons to sever electrical communication. The connecting tubing is blocked with a plug, with generation of two pressures. Each half contracts independently of the other, but for each half, contractions are synchronized with electrical activity. (C) Same configuration as in B, except the plug is removed, allowing hydraulic communication between halves, but electrical communication is disrupted. Synchronized activity among pressure and both electrodes is observed.

uterus contains, their location, whether or not they are fixed, of what cell types they are composed, and what the cellular events are that trigger action potentials.

In the heart-like model, the uterus has one electrogenic pacemaker at a fixed location, although data have consistently refuted this assumption. Caldeyro-Barcia's data demonstrated that there are at least two pacemakers located on each side of the fundus (Caldeyro-Barcia \& Poseiro 1959). He also showed that some contractions did not originate from the fundus (Caldeyro Barcia \& Alvarez 1952), although in his 'triple desending gradient' model, normal contractions only originate from one of the pacemakers in the fundus.

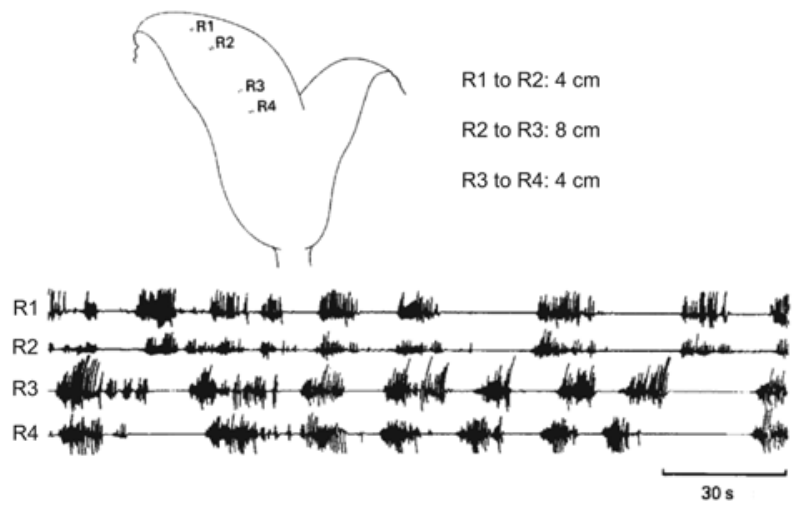

Figure 4 Uterine EMG recordings of pregnant ewe in labor, adapted from Parkington and coworkers (1988). R1 through R4 are pairs of recording electrodes sewn into the myometrium where indicated. Bioelectrical signals occasionally appear to be well coordinated, but there are no well-behaved waves that propagate long distances. The appearance of electrical signals is inconsistent with recruitment by action potential propagation.

After finding pacemaking activity at multiple sites in normal laboring patients, Csapo did not restrict the possible locations or suggest they are fixed (Csapo 1970b). He indicated that the activity of one pacemaker causes one local contraction, and there are several pacemakers in the uterus. But like Caldeyro-Barcia, he assumed that in labor, there is only one propagating contraction that is initiated by one pacemaker.

In 1971, Wolfs and coworkers (1971) used up to three pairs of EMG electrodes placed within the uterine cavity of near-term women while simultaneously measuring intrauterine pressure. Supporting the findings of Csapo, they noted that the location of the initial bioelectrical signal is not necessarily at the fundus, and additionally, the location varies from contraction to contraction in the same patient. Observing the progression of a patient from early labor to late labor, they noted that the bioelectrical signals 'synchronize' with pressure generation as labor progresses (Fig. 5). (The terminology may have been adapted from Csapo and Takeda (1963), who reported 'synchrony' of three uterine EMG signals at different sites in the rabbit.)

In their 1979 review, Wolfs and Van Leeuwen (1979) formulated a new model of organ-level function based largely on their own findings. They defined the 'contractile unit' as a localized contraction that results from the activity of one pacemaker, which is analogous to Csapo's 'local contraction'. However, in the Wolfs-van Leeuwen model, a coordinated contraction of labor results from simultaneous activity of many contractile units. This is significantly different from prior models since they proposed that multiple pacemakers are activated during a single contraction of labor. They clearly state that the mechanism for coordinating the contractile units is "open to speculation", but immediately follow this by arguing that coordination occurs via a unifying action 


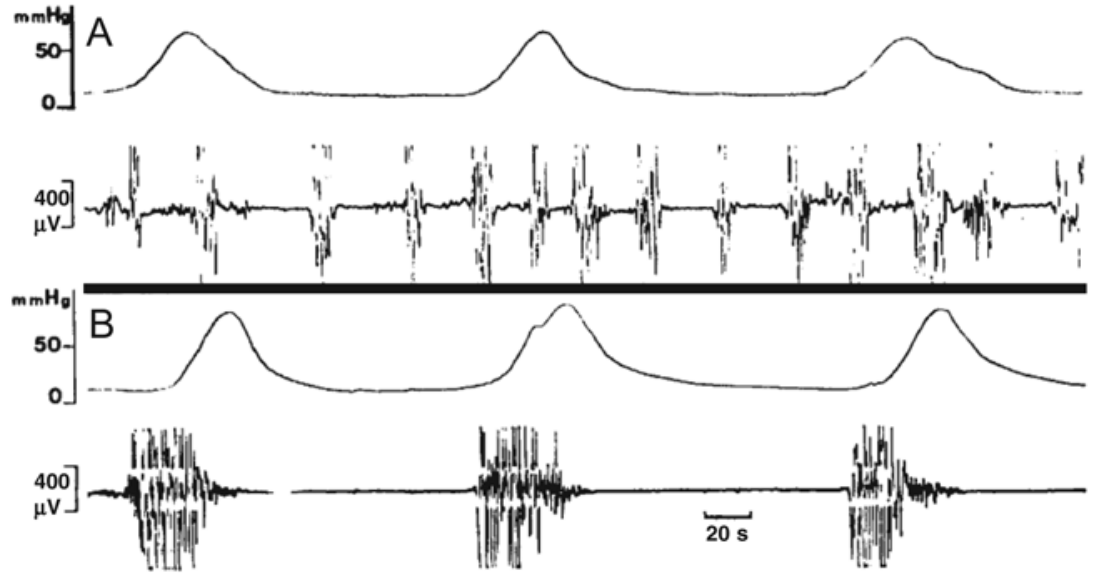

Figure 5 Simultaneous EMG and intrauterine pressure recordings, from Wolfs and coworkers (1971) and reproduced in Wolfs and Van Leeuwen (1979) with additional experimental description. The patient was undergoing mechanical induction. (A) Recordings made in early labor 100 min after inflation of an intrauterine balloon. Bioelectrical activity and pressure generation are 'unsynchronized'. (B) Five hours after the recordings in $(\mathrm{A})$, the patient is in advanced labor. There is a 1:1 correspondence between electrical signals and pressure. potential propagating throughout the uterus. Their support for a conventional action potential mechanism is not unexpected, since this paper was written immediately following the first report of uterine gap junctions (Garfield et al. 1977).

\section{Mechanical sensitivity of the pacemaker}

More experimental details appeared in Wolfs and van Leeuwen's 1979 review (Wolfs \& van Leeuwen 1979) describing how the 1971 data in Fig. 5 were obtained. This patient underwent balloon induction of labor for pre-eclampsia. While not explicitly stated, it is reasonable to assume that inflation of the balloon was intended to clinically initiate labor, and the patient was not experiencing significant contractions before induction. It was previously shown that foley balloon inflation ripens a closed cervix (Embrey \& Mollison 1967), but the links between mechanical stimulation, bioelectrical activity, and uterine contractions were problematic, and the data were not interpreted in this manner.

It is possible, however, to interpret these data based solely on hydrostatics and biomechanics. In this analysis, acutely inflating the balloon increases intrauterine volume and pressure. According to Laplace's Law, increased pressure increases wall tension, or stress, throughout the uterus. (Stress is tension per cross-sectional area.) The muscle elongates in response to increased stress, which drops pressure somewhat, but at a cost to wall thickness. As the uterine wall is not homogeneous, some areas of the wall experience more thinning relative to other areas, and the thinner areas will be more stressed. In summary, pressure and stress are greater at all areas of the uterine wall after balloon inflation than before, with some areas of the wall experiencing larger increases of stress than others.

About 100 min after balloon inflation (Fig. 5A), moderately coordinated contractions occurred every $3 \mathrm{~min}$, which generated moderate peak pressures $(55 \mathrm{mmHg})$. Relatively brief bioelectrical signals were also seen occuring at a very fast rate $(2-3 / \mathrm{min})$. This indicated that the electrode was sampling several contractile units (using Wolfs' terminology), or one contractile unit which was expressing a very high activity rate. The contractile unit, or units, generating these bioelectrical signals were not coordinated with the dominant contraction. Five hours later, the sampled contractile unit(s) coordinated with the others, and peak pressures increased to $80 \mathrm{mmHg}$ (Fig. 5B). Regardless of whether the electrode sampled one or more contractile units, the conclusion is that mechanical distention of the uterus greatly increased the activities of many pacemakers, and only a short time was required to create moderately coordinated contractions. Over a somewhat longer time, coordination of the pacemakers improved and peak pressures increased.

The observation that uterine distention increases uterine forces was previously reported by Csapo and coworkers, who transabdominally injected $500 \mathrm{~mL}$ isotonic solution directly into the uterine cavity. Csapo, of course, famously attributed the onset of labor to progesterone withdrawal, and explained these data based on the 'volume-to-progesterone ratio' (Csapo \& Lloyd-Jacob 1963). Thus, while both Csapo and Wolfs had evidence that over-distending the uterus enhances pacemaker activity, neither proposed that the mechanism was based on mechanotransduction, or that mechanical properties of the tissue actively influence contractions of labor.

In vitro isometric muscle bath experiments also support the concept that there are many uterine pacemakers. In these experiments, essentially, any small strip of uterine tissue obtained in mid or late pregnancy can spontaneously generate electrical activity and contractions. Each small tissue strip can, therefore, be said to contain an electrogenic pacemaker, and using this reasoning, a lower limit on the number of pacemakers in the entire uterus would, therefore, be in the thousands.

In the muscle bath, a small amount of tension must be placed on the tissue or it will not express pacemaker activity and contractions. This implies that electrogenic 
pacemakers are dependent on mechanical tension. To investigate the ability of mechanical force to initiate pacemaker activity and synchronize contractions, a twotissue experiment was performed (Young \& Goloman 2011) (Fig. 6). The tissues were fully isolated in separate experimental chambers, then mechanically linked endto-end with a frictionless bridge. Contact electrodes were placed on each tissue to record the timing of each tissue strip's electrical activity. Occasionally, the strips would coordinate contractile activities through the mechanical interactions, and express nearly synchronous electrical activity. As shown in the insert in Fig. 6, both electrical activity and force production of the "following" tissue begin after the onset of the tension caused by the "leading" tissue. Additionally, the electrical activity of the following tissue precedes its force production. This experiment demonstrates that tension caused by the leading tissue induced electrical activity in the following tissue, which resulted in contraction of the following tissue.

In summary, there is good support for the existence of multiple electrogenic pacemakers within the uterus, and that at least some are sensitive to mechanical stimulation. The question is, what synchronizes the pacemakers in laboring patients in vivo?

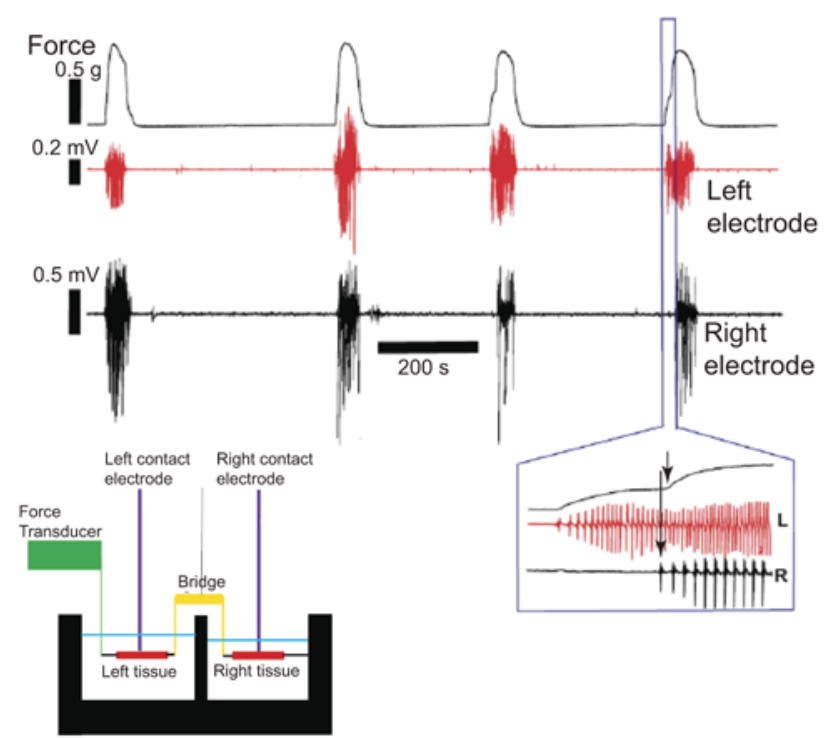

Figure 6 Two-tissue contractility experiment, adapted from Young and Goloman (2011). Two pregnant rat myometrial tissue strips placed in separate muscle baths, mechanically linked using a bridge. Force is measured simultaneously with electrical activities of each tissue to allow determination of when each tissue is active. Force: phasic force production is observed. Left electrode (red): bioelectrical signals from left tissue. Right electrode (black): bioelectrical signals from right tissue. Expansion (onset of last contraction): bioelectrical signals spontaneously appear from the left tissue, followed by onset of force. After a brief delay, bioelectrical signals appear from the right tissue, followed by further increasing force.

\section{Formulating the dual model}

The dual model is based on three premises (Young \& Barendse 2014). First, the localized bioelectrical activities observed in the SQUID array suggest that action potentials travel distances no more than $6-10 \mathrm{~cm}$, so long-distance propagation of one action potential is not the mechanism that coordinates uterine contractions at the level of the whole organ. Second, there are a number of mechanically sensitive electrogenic pacemakers distributed throughout the uterine wall, which initiate a number of tissue-level action potentials during each contraction. Third, coordinated activation of the pacemakers across long distances is accomplished using a mechanism involving intrauterine pressure, wall tension, and mechanotransduction.

The dual model can be summarized as follows (Fig. 7): If a localized contraction is expressed, the intrauterine pressure would increase slightly. Tension would increase proportionately and rapidly throughout the uterine wall because pressure is the same everywhere within an enclosed pressurized cavity (Pascal's principle). The higher tension would then recruit mechanically susceptible pacemakers regardless of physical location relative to the initial contraction. In this manner, a globally coordinated uterine contraction can be generated following a local event. This mechanism is similar to the hydrodynamic mechanism proposed by Takeda, but since bulk movement of fluid is not necessary, the mechanism is more correctly described as hydrostatic mechanotransduction.

\section{Step-wise description of the dual model (contractions of labor)}

1. The uterus is a hollow spheroid, filled with an incompressible fluid, and intrauterine pressure is on the order of $10 \mathrm{mmHg}$. Between contractions, there is little or no electrical or contractile uterine activity, although the baseline pressure places a baseline tension on the myometrium throughout the uterine wall.

2. An electrogenic pacemaker initiates an action potential at a random location within the uterine wall. Initiation could be spontaneous or mechanically induced.

3. A tissue-level action potential, or burst of action potentials, propagates $6-10 \mathrm{~cm}$ in $2-3 \mathrm{~s}$ and then terminates. Myometrium contracts when, and only when, it experiences an action potential. This local contraction is defined as a 'regional' contraction because it is limited to the region of the uterine wall that expresses one action potential event. Regions include the full thickness of the wall, and $6-10 \mathrm{~cm}$ in each of the two dimensions across the uterine surface. If the average-size is $8 \times 8 \mathrm{~cm}^{2}$, and assuming a spherical uterus containing $5 \mathrm{~L}$, the uterine wall is composed of approximately 22 regions.

4. The first regional contraction slightly raises the intrauterine pressure. 


\section{Dual model}
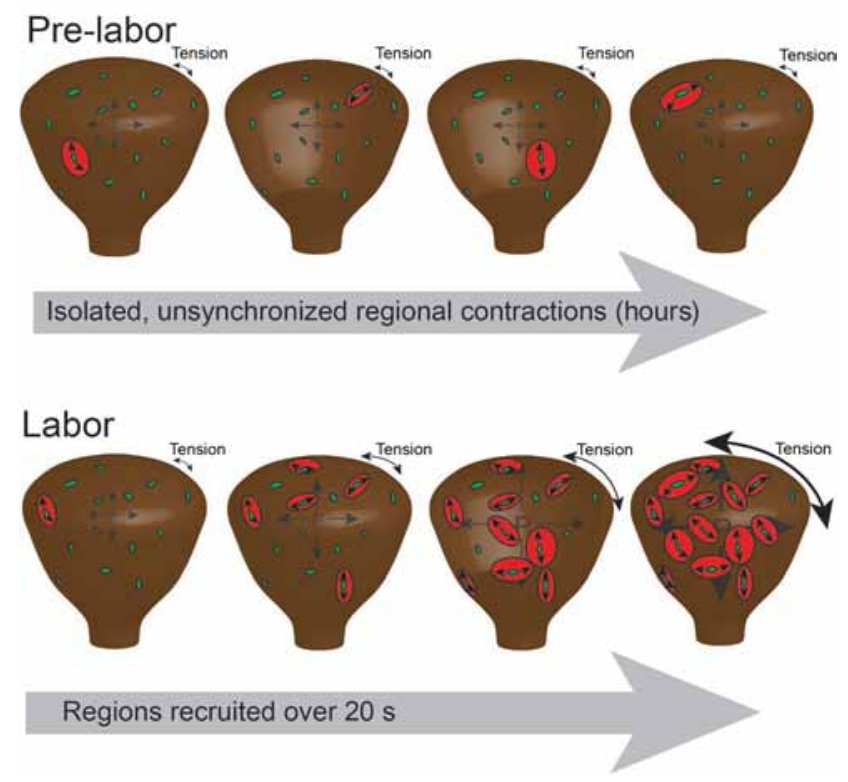

Figure 7 Dual model of pre-labor and labor contractions. Pre-labor: many potential sites of mechanosensitive pacemakers are distributed throughout the uterine wall (indicated by arbitrary location of green spots). In pre-labor, pacemakers are randomly and infrequently activated. Activation of a pacemaker initiates a propagating action potential (black arrow). The distance an action potential can propagate is limited, which results in a regional contraction (red). The first regional contraction slightly elevates intrauterine pressure $(\mathrm{P})$. Local wall tension increases throughout the uterine wall, but fails to mechanically activate other pacemakers. Labor: as with pre-labor, the contraction of the first region elevates intrauterine pressure and increases tension. The increased wall tension activates a second mechanically sensitive pacemaker and a second regional contraction, which further raises pressure. Higher pressure creates even greater wall tension, causing activation of more mechanically sensitive pacemakers in parallel. The feedback process continues until all mechanically sensitive pacemakers have activated most regions of the uterus at the same time.

5. The increased intrauterine pressure increases wall tension according to Laplace's Law; $T=P^{*} r / 2 w$, where $T$ is the local wall tension (technically stress), $P$ is the intrauterine pressure, $r$ is the local radius of curvature, and $w$ is the local wall thickness. Because of the dependency on local parameters, the amount of tension experienced at different locations will vary, even though the same intrauterine pressure is shared by all areas of the uterine wall.

6. A local increase in wall tension activates another mechanically sensitive pacemaker. There are no restrictions on the location of the new pacemaker site, since the wall tension increases throughout the uterus.

7. Activation of the newly recruited pacemaker initiates a propagating action potential, resulting in a regional contraction at the new location.

8. With contraction of another region, intrauterine pressure rises even more, wall tension throughout the uterus increases again, more mechanosensitive pacemakers are activated, and more regions are recruited to participate in the contraction. The process then repeats. This mechanism can be summarized as 'pressuretension-mechanotransduction'. Long-distance signaling is accomplished by the hydraulic (pressure-tension) components of the mechanism, while mechanotransduction recruits pacemakers.

9. This positive-feedback mechanism recruits regions in parallel, and provides a robust means to synchronize the activities of the mechanically sensitive pacemakers and coordinate the uterine contraction. The positive-feedback mechanism explains how the uterus repetitively generates consistently high intrauterine pressures during active labor.

10. Each region relaxes after a time determined by the metabolic and electrical properties of the tissue. After contracting, the tissue of a region enters a refractory period and then resets to become available for pressuretension-mechanotransduction recruitment and participation in the next contraction.

The relationship between wall tension and intrauterine pressure in steps 1-10 deviate slightly from Laplace's Law because it does not consider the following: (a) noncontracting regions elongate slightly with pressure rises and (b) the uterus becomes more spherical as intrauterine pressure rises. Elongation of noncontracting regions always tends to blunts pressure rises and slow pacemaker recruitment early in the contraction, but shape change creates more complex effects. Becoming more spherical decreases the surface area-to-volume ratio, which always tends to reduce pressure. However, changing shape may tend to either increase or decrease the local stress on a region depending on location. Between contractions, the front and back walls are relatively flat (large radius of curvature). When a contraction begins, these regions become more curved (the local radius of curvature decreases), which tends to decrease wall stress and slow pacemaker recruitment. The opposite occurs on the sides and fundus, which are more highly curved between contractions. As the contraction builds, deviations from Laplace's Law are reduced as a more spherical shape is achieved and the number of active regions begins to exceed the number of inactive regions. A comparison of the key elements of the dual model and the other models discussed in this paper are presented in Table 1.

\section{Transition into labor}

In addition to helping understand experimental data, a major goal of the dual model is to provide a framework to explain the transitions from quiescence, to prelabor, and then to labor. In the dual model, pre-labor contractions do not activate enough mechanically 
Table 1 Models for organ-level uterine function.

\begin{tabular}{|c|c|c|c|c|}
\hline Model name used in text & Long-distance signaling mechanism & Pacemaker & Functional unit of wall & Discrepant data \\
\hline Heart-like (1940s) & Action potential propagation (one) & One electrogenic; fixed location & None & $1,2,3$ \\
\hline Caldeyro-Barcia (1950) & $\begin{array}{l}\text { Myometrial contraction waves } \\
\text { (fundus to cervix) }\end{array}$ & $\begin{array}{l}\text { Unspecified type; one or two, } \\
\text { fixed locations in fundus }\end{array}$ & None & $1,2,3$ \\
\hline Takeda (1965) & Mechanotransduction & Unspecified type & Unspecified & 4 \\
\hline Csapo (1970) & $\begin{array}{l}\text { Action potential propagation (one } \\
\text { during labor) }\end{array}$ & $\begin{array}{l}\text { Electrogenic; not fixed; many } \\
\text { before labor, one in labor }\end{array}$ & Local contractions & 2 \\
\hline Wolfs-van Leeuwen (1979) & $\begin{array}{l}\text { Action potential propagation } \\
\text { (many, but one unifying) }\end{array}$ & $\begin{array}{l}\text { Electrogenic; not fixed; many } \\
\text { before and during labor }\end{array}$ & Contractile units & 5 \\
\hline Dual mechanism (2014) & $\begin{array}{l}\text { Pressure-tension- } \\
\text { mechanotransduction }\end{array}$ & $\begin{array}{l}\text { One mechanically sensitive } \\
\text { electrogenic pacemaker for } \\
\text { each region, many regions }\end{array}$ & Regions & 6 \\
\hline
\end{tabular}

1. Strong evidence against fixed pacemaker; 2 . Many propagating action potentials observed within one contraction; 3 . No preferred direction of propagation of bioelectrical activity; 4 . Action potentials precede pressure rises; 5 . No evidence for unifying action potential; 6 . Needs further direct testing.

sensitive pacemakers to enter the positive-feedback phase for recruitment of regions (steps 6-10). This suggests that the uterus transitions into a labor phenotype by going through the following phases:

1. expression of the cellular components required for membrane excitability and excitation-contraction coupling, including electrogenic pacemaker activity;

2. expression of connexin 43 to allow coupling of individual myocytes into a tissue-level syncytium, and the ability to propagate tissue-level action potentials over distances of $6-10 \mathrm{~cm}$;

3. expression of mechanical sensitivity of the electrogenic pacemakers.

The transition from quiescence to pre-labor is progressively accomplished through phases 1 and 2 . The onset of labor occurs with phase 3 , and emphasizes the clinical importance of mechanotransduction. Because contractions of labor utilize an entirely different signaling mechanism, the dual model identifies contractions of pre-labor and contractions of labor as fundamentally different.

\section{Resolving discrepancies of data on long-distance signaling in the context of the dual model}

As detailed in the section 'Problems with action potential propagation as a mechanism for tissue recruitment over long distances', empirical data do not easily support an action potential propagation mechanism for long-distance signaling. The three key problems are summarized here, and interpreted in the context of the dual model: First, there are limits on the distances action potentials can propagate. The dual model accepts that action potentials terminate after propagating $\sim 6-10 \mathrm{~cm}$. This distance establishes the size of each region. Second, action potential propagation is slow and tortuous, and would require long times to activate the entire uterus. In the dual model, action potential propagation recruits tissue only within regions, not over long distances. Because regions are small, pathways can be tortuous and still require less than a few seconds to complete tissue recruitment. Third, uterine bioelectrical activities arise randomly, at distant sites, and without continuous propagation pathways. Pressure-tension signaling occurs rapidly throughout the entire uterine wall regardless of the physical location of the regions previously activated. Recruitment is not restricted to near-neighbor interactions. Once a contraction is initiated, regions are recruited in parallel rather than in series or contiguously. There are no necessary or preferred directions for action potential propagation within regions because there is no near-neighbor signaling requirement. Hydraulic signaling requires only a fraction of a second to travel to all parts of the uterine wall. The mechanotransduction step is rate limiting, and depends on the mechanical sensitivity of each pacemaker.

\section{Clinical implications pressure-tension- mechanotransduction}

Intrauterine pressure is a product of uterine contractions in all models, but in the dual model, intrauterine pressure is uniquely a key intermediary in the mechanism of long-distance signaling. Hence, the dual model anticipates that any clinical variable that affects pressure or local wall tension will also affect the expression of labor. From Laplace's Law, the parameters are resting pressure, local wall thickness, and the local radius of curvature. As examples, local wall tension would increase with iatrogenic uterine over-distention (Csapo et al. 1963, Wolfs et al. 1971) (increased resting pressure, thinning of wall), chronic over-distention resulting from hydramnios or multiple gestation (thin uterine wall), and uterine anomalies or myomas distorting the wall (large or irregular local radius of curvature). Interestingly, these clinical conditions are also associated with preterm labor, suggesting that myometrial biomechanics may directly relate to abnormal labor.

\section{Research areas underrepresented}

It is clearly important to understand all aspects of uterine contractility, butgoing forward, it is reasonable to emphasize 
areas that previously have been understudied. Specifically, there has been a lack of emphasis on myometrial mechanical properties and mechanotransduction, largely because it has not been fully appreciated how these areas are relevant to normal and abnormal labor. If organ-level coordination is to be understood, the study of the mechanical sensitivity of pacemaker activity also seems necessary.

Finally, one of the goals of an organ-level model is to provide a framework for predicting clinical effects based on changing cellular events. The dual model proposes that regions are functional intermediaries residing between cell and organ function, and that they need to be considered when assessing the emergent properties of an unstable system (Banney et al. 2015). The dual model offers a uniting framework that provides an opportunity to further incorporate both novel and discarded concepts, with a goal of identifying new methods to evaluate and treat abnormal labor.

\section{Declaration of interest}

Roger C Young holds financial interest in PreTel, Inc., which is a medical device company developing new methods to measure uterine contractions in labor.

\section{Funding}

This research did not receive any specific grant from any funding agency in the public, commercial or not-for-profit sector.

\section{Acknowledgements}

The author is grateful for the constructive input of Dr Wim Lammers and Dr Brynjar Karlsson during manuscript preparation.

\section{References}

Alvarez H \& Caldeyro R 1950 Contractility of the human uterus recorded by new methods. Surgery, Gynecology \& Obstetrics 91 1-13.

Banney D, Young RC, Paul JW, Imtiaz M \& Smith R 2015 A hypothesis for self-organization and symmetry reduction in the synchronization of organ-level contractions in the human uterus during labor. Symmetry 7 1981-1988. (doi:10.3390/sym7041981)

Barclay M, Andersen H \& Simon C 2010 Emergent behaviors in a deterministic model of the human uterus. Reproductive Sciences $\mathbf{1 7}$ 948-954. (doi:10.1177/1933719110376544)

Bozler E 1947 The response of smooth muscle to stretch. American Journal of Physiology 149 299-301.

Caldeyro-Barcia R \& Alvarez H 1952 Abnormal uterine action in labor. British Journal of Obstetrics and Gynecology 59 646-656.

Caldeyro-Barcia R \& Poseiro JJ 1959 Oxytocin and contractility of the pregnant human uterus. Annals of the New York Academy of Sciences 75 813-830. (doi:10.1111/nyas.1959.75.issue-2)

Caldeyro-Barcia R, Sica-Blanco Y, Poseiro J, Gonzalez Panizza V, Mendez-Bauer C, Fielitz C, Alvarez H, Pose SV \& Hendricks CH 1957 A quantitative study of the action of synthetic oxytocin on the pregnant human uterus. Journal of Pharmacology and Experimental Therapeutics 121 18-31.

Csapo A 1959 Studies on excitation-contraction coupling. Annals of the New York Academy of Sciences 81 453-467. (doi:10.1111/j.1749-6632. 1959.tb49327.x)
Csapo A 1970a The diagnostic significance of the intrauterine pressure. I. Obstetrical \& Gynecological Survey 25 403-435.

Csapo A $1970 b$ The diagnostic significance of the intrauterine pressure. II. Clinical considerations and trials. Obstetrical \& Gynecological Survey 25 515-543.

Csapo Al \& Lloyd-Jacob MA 1963 Effect of uterine volume on parturition. American Journal of Obstetrics and Gynecology 85 806-812.

Csapo A \& Suzuki T 1957 A preliminary note on excitation-contraction coupling. PNAS 43 278-281. (doi:10.1073/pnas.43.3.278)

Csapo Al \& Takeda H 1963 Electrical activity of the parturient human uterus. Nature 200 680-682. (doi:10.1038/200680a0)

Csapo Al \& Takeda H 1965 Effect of progesterone on the electric activity and intrauterine pressure of pregnant and parturient rabbits. American Journal of Obstetrics and Gynecology 91 221-231. (doi:10.1016/00029378(65)90204-8)

Csapo Al, Jaffin H, Kerenyi T, Lipman JI \& Wood C 1963 Volume and activity of the pregnant human uterus. American Journal of Obstetrics and Gynecology 85 819-835.

Embrey MP \& Mollison BG 1967 The unfavourable cervix and induction of labour using a cervical balloon. Journal of Obstetrics and Gynaecology of the British Commonwealth 74 44-48. (doi:10.1111/bjo.1967.74.issue-1)

Garfield RE, Sims S \& Daniel EE 1977 Gap junctions: their presence and necessity in myometrium during parturition. Science 198 958-960. (doi:10.1126/science.929182)

Garfield RE, Ali M, Yallampalli C \& Izumi H 1995 Role of gap junctions and nitric oxide in control of myometrial contractility. Seminars in Perinatology 19 41-51.

Jeffcoate TN 1948 Incoordinate uterine action in labour. Canadian Medical Association Journal 58 42-48.

Lammers WJ 2013 The electrical activities of the uterus during pregnancy. Reproductive Sciences 20 182-189. (doi:10.1177/1933719112446082)

Lammers WJ, Mirghani H, Stephen B, Dhanasekaran S, Wahab A, Al Sultan MA \& Abazer F 2008 Patterns of electrical propagation in the intact pregnant guinea pig uterus. American Journal of Physiology. Regulatory, Integrative and Comparative Physiology 294 R919-R928. (doi:10.1152/ajpreggu.00704.2007)

Lucovnik M, Maner WL, Chambliss LR, Blumrick R, Balducci J, NovakAntolic Z \& Garfield RE 2011 Noninvasive uterine electromyography for prediction of preterm delivery. American Journal of Obstetrics and Gynecology 204228 e221-e210. (doi:10.1016/j.ajog.2010.09.024)

Mikkelsen E, Johansen P, Fuglsang-Frederiksen A \& Uldbjerg N 2013 Electrohysterography of labor contractions: propagation velocity and direction. Acta Obstetricia et Gynecologica Scandinavica 92 1070-1078. (doi:10.1111/aogs.12190)

Mironneau J 1973 Excitation-contraction coupling in voltage clamped uterine smooth muscle. Journal of Physiology 233 127-141. (doi:10.1113/ jphysiol.1973.sp010301)

Parkington HC, Harding R \& Sigger JN 1988 Co-ordination of electrical activity in the myometrium of pregnant ewes. Journal of Reproduction and Fertility 82 697-705. (doi:10.1530/jrf.0.0820697)

Rabotti C \& Mischi M 2015 Propagation of electrical activity in uterine muscle during pregnancy: a review. Acta Physiologica 213 406-416. (doi:10.1111/apha.12424)

Rabotti C, Mischi M, Oei SG \& Bergmans JW 2010 Noninvasive estimation of the electrohysterographic action-potential conduction velocity. IEEE Transactions on Bio-Medical Engineering 57 2178-2187. (doi:10.1109/ TBME.2010.2049111)

Rabotti C, Oei SG, van ‘t Hooft J \& Mischi M 2011 Electrohysterographic propagation velocity for preterm delivery prediction. American Journal of Obstetrics and Gynecology 205 e9-e10. (doi:10.1016/j. ajog.2011.06.071)

Ramon C, PreissI H, Murphy P, Wilson JD, Lowery C \& Eswaran H 2005 Synchronization analysis of the uterine magnetic activity during contractions. Biomedical Engineering Online 4 55. (doi:10.1186/1475-925X-4-55)

Sigger JN, Harding R \& Jenkin G 1984 Relationship between electrical activity of the uterus and surgically isolated myometrium in the pregnant and nonpregnant ewe. Journal of Reproduction and Fertility 70 103-114. (doi:10.1530/jrf.0.0700103)

Smith R, Imtiaz M, Banney D, Paul JW \& Young RC 2015 Why the heart is like an orchestra and the uterus is like a soccer crowd. American Journal of Obstetrics and Gynecology 213 181-185. (doi:10.1016/j. ajog.2015.06.040) 
Takeda H 1965 Generation and propagation of uterine activity in situ Fertility and Sterility 16 113-119. (doi:10.1016/S0015-0282(16)35473-5)

Wolfs GM \& van Leeuwen M 1979 Electromyographic observations on the human uterus during labour. Acta Obstetricia et Gynecologica Scandinavica. Supplement 90 1-61. (doi:10.3109/ 00016347909156375 )

Wolfs G, van Leeuwen M, Rottinghuis H \& Boeles JT 1971 An electromyographic study of the human uterus during labor. Obstetrics and Gynecology 37 241-246.

Wray S, Burdyga T, Noble D, Noble K, Borysova L \& Arrowsmith S 2015 Progress in understanding electro-mechanical signalling in the myometrium. Acta Physiologica 213 417-431. (doi:10.1111/apha.12431)

Young RC 2015 Synchronization of regional contractions of human labor; direct effects of region size and tissue excitability. Journal of Biomechanics 48 1614-1619. (doi:10.1016/j.jbiomech.2015.02.002)
Young RC \& Barendse P 2014 Linking myometrial physiology to intrauterine pressure; how tissue-level contractions create uterine contractions of labor. PLoS Computational Biology 10 e1003850. (doi:10.1371/journal. pcbi.1003850)

Young RC \& Goloman G 2011 Mechanotransduction in rat myometrium: coordination of contractions of electrically and chemically isolated tissues. Reproductive Sciences 18 64-69. (doi:10.1177/1933719110379637)

Received 22 March 2016

First decision 5 April 2016

Revised manuscript received 24 April 2016

Accepted 9 May 2016 